\title{
Artículo de Investigación \\ Enfermedad de Hailey- Hailey: reporte de casos entre los años 2001 y 2016 en un hospital universitario y revisión de la literatura
}

\author{
Ximena Chaparro ${ }^{1,2}$, Viera Kaplan ${ }^{3}$, Constanza Pulgar ${ }^{4}$, Tirsa Saavedra $^{5}$, Claudia Morales ${ }^{6}$, Laura Carreño ${ }^{6}$
}

\section{RESUMEN}

Introducción: La enfermedad de Hailey-Hailey (pénfigo crónico familiar benigno) es una dermatosis ampollar, de evolución crónica, recidivante, autosómica dominante, de penetrancia y expresividad variables, localizada predominantemente en los pliegues.

Métodos: Se realizó una revisión de la base de datos del Servicio Anatomía Patológica del Hospital Clínico Universidad de Chile (HCUCH) entre los años 2001 y 2016 y se complementaron con los antecedentes clínicos extraídos de las fichas clínicas.

Resultados: Se obtuvo un total de 6 casos ilustrativos de la patología en estudio.

Discusión y conclusiones: La enfermedad de Hailey-Hailey es una genodermatosis poco frecuente cuyo diagnóstico y tratamiento constituyen un desafío para el dermatólogo.

Palabras clave: Enfermedad de Hailey-Hailey; Pénfigo crónico familiar benigno; Alteración acantolítica, genodermatosis.

\section{SUMMARY}

Background: The Hailey-Hailey disease (familial benign chronic pemphigus) is a bullous dermatosis, with chronic and recurrent evolution, autosomal dominant with variable penetrance and expressivity, localized predominantly in the folds.

Methods: A review of the database of the Anatomic Pathology Service of the Hospital Clínico Universidad de Chile (HCUCH) between 2001 and 2016 was carried out and supplemented with the clinical history extracted from the clinical records.

Results: A total of 6 illustrative cases of the pathology under study were obtained.

Discussion and conclusions: Hailey-Hailey's disease is a rare genodermatosis whose diagnosis and treatment is a challenge for the dermatologist.

Key words: Hailey-Hailey disease; familial benign chronic pemphigus; Acantholytic disorder, genodermatosis.
$\mathrm{L}$ a enfermedad de Hailey-Hailey (EHH), también llamada pénfigo crónico familiar benigno, es un trastorno acantolítico con herencia autosómica dominante, poco frecuente, caracterizada por vesículas y erosiones recurrentes en zonas de pliegues. ${ }^{1,2}$

Su diagnóstico suele ser tardío por confundirse con otras patologías como eccema, infecciones bacterianas o micóticas.

\footnotetext{
${ }^{1}$ Unidad de Dermatología Infantil, Servicio de Pediatría Hospital Dr. Exequiel González Cortés, Santiago, Chile. 'Servicio de Dermatología, Departamento de Cirugía, Clínica Alemana de Santiago, Facultad de Medicina CAS-UDD, Santiago, Chile. ${ }^{3}$ Departamento Dermatología, Facultad de Medicina, Universidad de Chile, Santiago, Chile ${ }^{4}$ Servicio de Dermatología, Hospital Clínico San Borja Arriarán, Santiago, Chile. ${ }^{5}$ Servicio de Dermatología, Clínica Santa María, Santiago, Chile. ${ }^{6}$ Servicio de Anatomía Patológica, Hospital Clínico de la Universidad de Chile, Santiago, Chile
}

Correspondencia: Viera Kaplan Z Correo electrónico: vierakaplan@ gmail.com Teléfono: +56 968294682 Dirección: Santos Dumont 999, Independencia, Santiago, Chile. Código Postal: 8380456.
No existen tratamientos específicos ni totalmente efectivos. Se utilizan corticoides y antibióticos tópicos, terapias sistémicas, quirúrgicas y físicas.

\section{Materiales y MÉTodos}

Con el objetivo de revisar los casos de EHH diagnosticados y tratados en el HCUCH en los últimos años, se realizó una revisión de la base de datos del Servicio Anatomía Patológica del Hospital Clínico Universidad de Chile (HCUCH) entre los años 2001 y 2016 y se complementaron con los antecedentes clínicos extraídos de las fichas clínicas.

\section{Resultados}

Se registraron un total de 6 pacientes con enfermedad de Hailey- Hailey. Éstos se describen continuación: 
CAso 1: Mujer de 46 años con antecedente de dermatitis atópica del adulto. Presenta historia de 3 años de evolución de placas eritematosas y descamativas en fosas poplíteas y pliegues antecubitales, axilas, región intermamaria y zona cervical, asociado a prurito y ardor. Presenta una hija con afección similar.(Figura la)

Se realiza biopsia de piel a placa región intermamaria, en donde se informa una dermatitis vesiculo bulosa intraepidérmica suprabasal acantolítica, compatible con Enfermedad de Hailey- Hailey. (Figura 1b)

Se maneja con corticoides de alta potencia más antibióticos tópicos con buena respuesta clínica. Su hija se realiza biopsia de las lesiones en donde también se confirma Enfermedad de Hailey Hailey.

CAso 2: Hombre de 51 años, sin antecedentes mórbidos. Presenta desde los 30 años, eritema, irritación y secreción serosa de mal olor en axilas, pliegue interglúteo, escroto y pliegue abdominal. Es tratado en múltiples ocasiones como intertrigo micótico, dermatitis de contacto sobreinfectada, sin buena respuesta a los tratamientos. (Figura 2a)

Se tomó biopsia de la piel de una de las placas axilares que mostró una dermatosis vesico-ampollosa intraepidérmica, de predominio suprabasal, compatible con Enfermedad de Hailey Hailey.(Figura 2b)

Es manejado con Dapsona, con excelente respuesta.

Dentro de la historia clínica destacaba que su madre y tía materna sufren afección similar, en donde se confirma por medio de biopsia Enfermedad de Hailey Hailey.

CAso 3: Hombre de 61 años, con antecedentes de hipertensión arterial. Presenta un cuadro de 10 años de evolución de ampollas en región inguinal, axilar y cervical, que se rompen y dejan la zona erosionada, formando posteriormente placas eritematosas, algo hipertróficas, de mal olor. (Figura 3a) 2001 y 2016.
Se realiza biopsia, que muestra una dermatitis vesículobulosa intraepidérmica suprabasal acantolítica compatibles con Enfermedad de Hailey Hailey.(Figura 3b)

En la historia clínica destaca madre y hermano con Enfermedad de Hailey Hailey confirmada por biopsia.

Es tratado con corticoides tópicos de alta potencia con buena respuesta.
La enfermedad de Hailey-Hailey es una dermatosis ampollar, de evolución crónica, recidivante, autosómica domiexpresividad te en los pliegues.

Dado que es una genodermartosis poco frecuente, la presente investigación permite describir y analizar los casos vistos en un hospital universitario en el periodo comprendido entre el año

Se pretende recordar los aspectos para su diagnóstico y traentregando herramientas al dermatólogo para su manejo adecuado.
CAso 4: Mujer de 47 años, con antecedentes de intolerancia a la glucosa y alergia a las castaña de indias.

Presenta historia de 2 años de evolución placas eritematosas y rezumantes, pruriginosas y en ocasiones dolorosas en región vulvar, vaginal y perianal. Utilizó múltiples tratamiento tópicos sin respuesta. (Figura $4 \mathrm{a}$ y $4 \mathrm{~b})$

Se realiza biopsia de piel vulvar e inguinal que muestra piel con acantolisis intraepidérmica de predominio suprabasal con células de citoplasma eosinófilo redondeado. Infiltrado inflamatorio dérmico con linfocitos, macrófagos y escasos eosinófilos.(Figura 4c)

CAso 5: Mujer de 56 años con antecedente de padre y abuelo con Enfermedad de Hailey-Hailey presenta pápulas eritematosas que confluyen en placas erosionadas costrosas de aspecto vegetante pruriginosas en cuero cabelludo, cuello, fosas antecubitales y poplíteas. (Figura 5 a y $5 b)$

Se realiza biopsia de cuero cabelludo y que muestra epidermis con hendiduras suprabasales con células acantolíticas y aislados disqueratocitos, dermis con infiltrado perivascular superficial e intersticial linfocitario con polimorfonucleares y eosinófilos.(Figura 5c)

CAso 6: Mujer de 64 años, hipertensa, con historia de 1 año de evolución de lesiones pruriginosas y de mal olor en ambos pliegues inguinales. Ha recibido tratamiento 

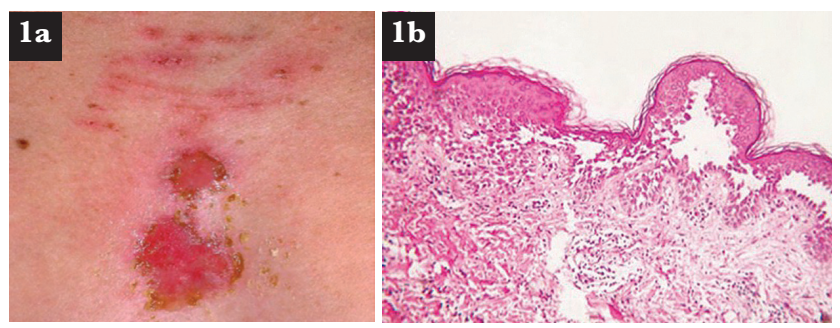

\section{Figuras}

1a. Enfermedad de Hailey- Hailey: Placas erosivas de 1-2 cm de diámetro, ovaladas, de límites netos con costras melicéricas, superficie exudativa, brillante, con pequeñas vesículas a nivel central, sobre una base eritematosa, en la región intermamaria.

1b. Enfermedad de Hailey- Hailey: HE 40x. Ampolla intraepidérmica suprabasal, cuyo techo está formado por varias hileras de queratinocitos unidos parcialmente, que determinan una imagen típica de "pared de ladrillos derrumbados".
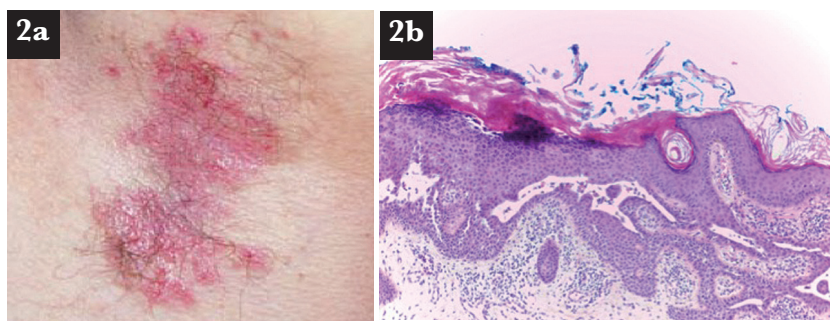

\section{Figuras}

2a. Enfermedad de Hailey- Hailey: Placa eritematosa, húmeda con erosiones lineales en su superficie.

2b. Enfermedad de Hailey- Hailey: HE 100x. Hendidura acantolítica disqueratótica intraepidérmica suprabasal.
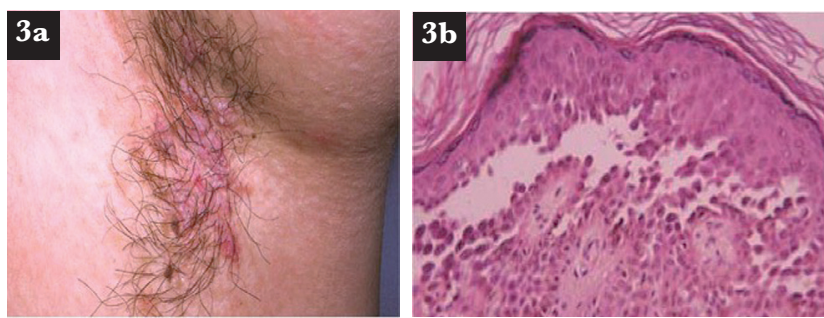

\section{Figuras}

3a. Enfermedad de Hailey- Hailey: Placas de aspecto hipertrófico, eritematosas, maceradas, localizadas en la axila izquierda.

3b. Enfermedad de Hailey- Hailey: HE 20x. Acantolisis preferentemente suprabasal.
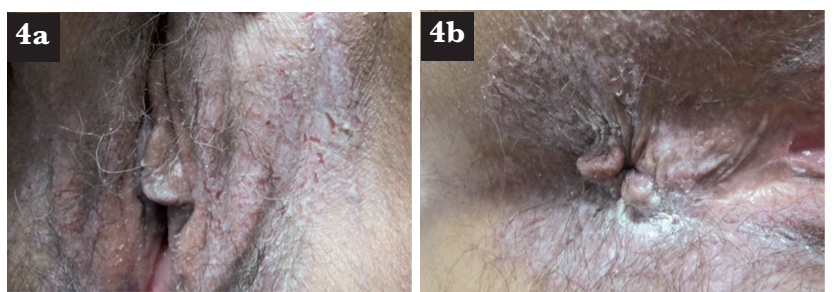

\section{Figuras}

4a. Enfermedad de Hailey- Hailey: Placa violácea macerada que compromete el pliegue inguinal y el labio mayor.

4b. Enfermedad de Hailey- Hailey: Placa violácea macerada que compromete la zona perianal.

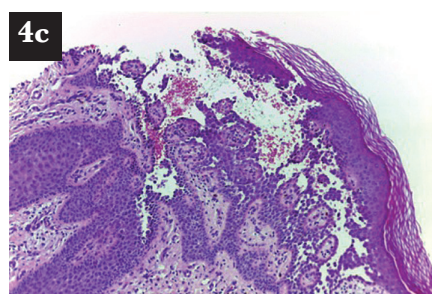

\section{Figura 4c}

Enfermedad de Hailey- Hailey: HE 40x. Piel con lesión acantolítica intraepidérmica suprabasal con escasa inflamación
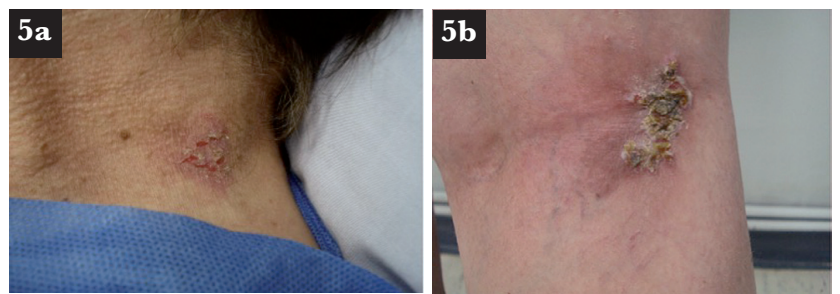

\section{Figuras}

5a. Enfermedad de Hailey- Hailey: Placa erosionada costrosas de aspecto vegetante en cuello lateral izquierdo

5b. Enfermedad de Hailey- Hailey: Placa costrosa sobre base eritematosa en fosa poplítea derecha.

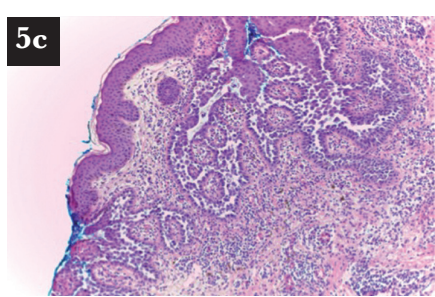

\section{Figura 5c}

Enfermedad de Hailey- Hailey: HE 100x. Lesión acantolítica disqueratótica intraepidérmica suprabasal. Dermis con linfocitos y eosinófilos.
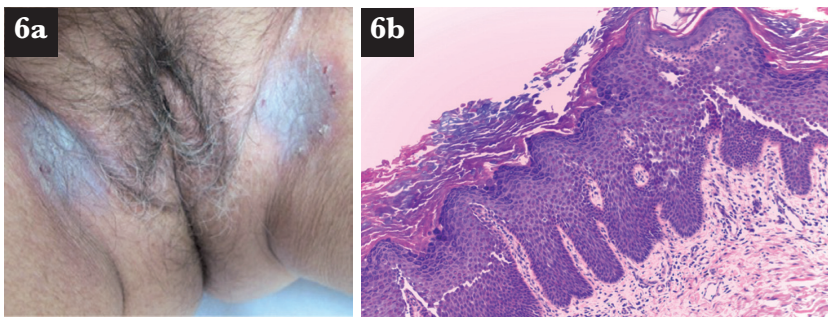

Figuras

6a. Enfermedad de Hailey- Hailey: Placas violáceas de aspecto macerado y verrucoso con zonas erosionadas en región inguinal bilateral.

6b. Enfermedad de Hailey- Hailey: HE 100x. Dermatitis acantolítica, disqueratótica intraepidérmica. 
antimicótico en múltiples ocasiones con resultado parcial. Al examen destacan placas violáceas de superficie verrucosa blanquecina en ambos pliegues inguinales. (Figura 6a)

Se indican medidas generales, bifonazol 1\% tópico y solicitan exámenes generales y test de parche. $\mathrm{Al}$ mes, con resultado de test de parche negativo y escasa respuesta al manejo tópico se decide se solicitar biopsia con inmunofluorescencia directa por sospecha de enfermedad de Hailey- Hailey versus pénfigo vegetante.

Biopsia informa epidermis con acantosis e hiperqueratosis leve acantolisis y células disqueratóticas intraepidérmicas de predominio en tercio basal, inmunofluorescencia directa negativa. Hallazgos morfológicos e inmunofluorescencia concordantes con enfermedad de Hailey- Hailey (Figura 6b) (Tabla 1)

\section{Discusión}

La EHH es una genodermatosis de herencia autosómica dominante con penetrancia completa que se presenta entre la tercera y cuarta década de la vida. ${ }^{1}$ Sin embargo la edad de aparición y expresividad varían en forma considerable entre los miembros afectados de una familia.

$\mathrm{Su}$ etiopatogenia no es bien conocida, se postula que existiría una mutación del gen ATP2G1 localizado en el cromosoma 3q21-q24 que codifica para la proteína hsP-CA1la, la cual regula el flujo de Ca2+ entre el aparato de Golgi y el citosol. ${ }^{2}$ Esto produce una alteración en el procesamiento de las proteínas desmosomales y una transición anormal de la queratina 14 a la 10, lo que resulta en la acantolisis del estrato espinoso. Se postula que existen otras bombas dependientes de ATP que pueden influir en la expresividad de la $\mathrm{EHH}$, generando presentaciones atípicas de la enfermedad. ${ }^{3}$

La lesión inicial consiste en una vesícula flácida sobre piel normal o eritematosa, que se rompe fácilmente. El signo de Nikolsky es, a menudo, positivo en las áreas afectadas. Evolucionan en brotes desarrollando placas que se extienden por la periferia con un borde circinado. En el centro de la lesión se forman vesículas pequeñas, que se rompen dejando una superficie erosiva y exudativa que evoluciona a escamo costras crónicas, húmedas y de mal olor con fisuras dolorosas. ${ }^{6}$ Se localizan principalmente a nivel de los pliegues cutáneos, como la ingle, los surcos submamarios, las caras laterales del cuello y la axila, con menos frecuencia el cuero cabelludo, fosas antecubitales y poplíteas. ${ }^{4}$ Las lesiones curan sin dejar cicatrices, pudiendo existir hiperpigmentación residual.

El proceso es benigno, pero se acompaña de dolor, prurito y mal olor en muchos de los casos. ${ }^{5}$ Esto último fue manifestado por la mitad de los pacientes reportados en este estudio.

Se han identificado factores desencadenantes como fricción, exposición solar, calor, sudoración, frío y agentes infecciosos.

El diagnóstico definitivo está dado por el estudio histológico de la piel comprometida. Se describe formación de hendiduras o ampollas intraepidérmicas secundarias a acantólisis suprabasal. La acantolisis suele ser extensa y afecta a varias capas del estrato espinoso.

Tabla 1. Resumen de los resultados. Edad expresada en años. Tiempo de evolución de la enfermedad en años. Zonas comprometidas expresada en números. X: dato no consignado en la ficha clínica.

\begin{tabular}{|c|c|c|c|c|c|c|}
\hline Variables & Caso 1 & Caso 2 & Caso 3 & Caso 4 & Caso 5 & Caso 6 \\
\hline Sexo & $\mathrm{F}$ & $\mathrm{H}$ & $\mathrm{H}$ & $\mathrm{M}$ & $\mathrm{M}$ & $\mathrm{M}$ \\
\hline Edad & 46 & 51 & 61 & 47 & 56 & 64 \\
\hline Antecedente familiar & Hija & Madre y tía & Madre y hermano & No & Padre y abuelo & No \\
\hline Años de evolución & 3 & 21 & 10 & 2 & $\mathrm{x}$ & 1 \\
\hline Sintomatología & Prurito- ardor & $\begin{array}{l}\text { Secreción } \\
\text { maloliente }\end{array}$ & $\begin{array}{c}\text { Lesiones } \\
\text { malolientes }\end{array}$ & Prurito & $\mathrm{x}$ & $\begin{array}{l}\text { Prurito y } \\
\text { secreción } \\
\text { maloliente }\end{array}$ \\
\hline Zonas comprometidas & 5 & 4 & 3 & 3 & 4 & 1 \\
\hline Terapia & Tópica & Sistémica & Tópica & Tópica & $\mathrm{x}$ & $\mathrm{x}$ \\
\hline Evolución & Favorable & Favorable & Favorable & Escasa respuesta & $\mathrm{x}$ & $\mathrm{x}$ \\
\hline
\end{tabular}


El suelo de la ampolla suele ser una fina capa de células basales sobre la que se adhieren queratinocitos parcialmente inconexos que dan un aspecto comparable a "s". Las papilas dérmicas, cubiertas por células basales parecen un muro de ladrillos derrumbado con profusión en las ampollas como vellosidades en "dedo de guante".?

La inmunofluorescencia directa e indirecta son siempre negativas, a diferencia de las enfermedades ampollares autoinmunes. La microscopia electrónica muestra un defecto en la adhesión desmosómica con separación de los tonofilamentos.

Presenta múltiples diagnósticos diferenciales de distinta índole, tanto infecciosas como inflamatorias y ampollares, a saber destacan la Enfermedad de Darier, el Pénfigo vulgar variedad vegetante, la Dermatitis seborreica, la Psoriasis inversa, el Intértrigo candidiásico, Erityrasma y la Paraqueratosis granular. Es habitual que el diagnóstico sea más bien tardío por la baja prevalencia de la enfermedad y su similitud con varias patologías dermatologicas más frecuentes, la media en nuestro estudio fue de 7,4 años entre los 5 pacientes en que se consignó el tiempo de evolución.

No existe un tratamiento específico para la EHH. La evidencia de la eficacia de las terapias tópicas o sistémicas se limita a los informes de casos individuales o pequeñas series de casos. El abordaje terapéutico de EHH implica el control de los factores que la exacerban, infecciones secundarias y la inflamación cutánea. Su manejo específico es complejo, incluye antibióticos y corticoides, tópicos y sistémicos. El rol de antibióticos sistémicos a bajas dosis por tiempo prolongado, ha demostrado disminuir las recurrencias asociado a su efecto antiinflamatorio. ${ }^{8}$ La dapsona se menciona en la literatura como efectiva en dosis de 50 a $250 \mathrm{mg} /$ día, sin embargo, no hay estudios recientes que avalen su efectividad.

De los 6 casos estudiados, fue posible rescatar el tratamiento llevado a cabo en 4, de los cuales 3 recibieron terapia tópica, corticoides asociados o no a antibióticos. La evolución fue favorable en 2 de los 3 casos. Se indicó tratamiento sistémico en 1 caso, se utilizó Dapsona sistémica con buena respuesta a pesar de la escasa y antigua evidencia que la respalda. Hacen falta estudios actuales que permitan respaldar su utilidad en el pénfigo familiar.

En casos recalcitrantes otras terapias sistémicas que han sido reportadas como beneficiosas incluyen el uso de inmunosupresores como ciclosporina, ${ }^{9}$ toxina botulínica A y anticolinérgicos sistémicos como el glicopirrolato. Éstos últimos actúan inhibiendo la producción de sudor y secundariamente disminuyendo la colonización microbiana. El glicopirrolato es un agente anticolinérgico sistémico de vida media corta con menos efectos adversos anticolinérgicos relacionados con el sistema nervioso central. $^{10,11,12}$

Se han reportado casos con buena respuesta a la radioterapia con electron beam, el que dirigido a una profundidad de 2 a 2,5 $\mathrm{mm}$ actuaría generando perturbación de la función intracelular, inmunosupresión e inhibición de la proliferación celular epidérmica excesiva. Se han descrito periodos libres de síntomas hasta por 28 meses. Sin embargo los resultados de otros reportes han sido contradictorios. ${ }^{13}$

En casos refractarios, se pueden realizar procedimientos quirúrgicos y ablativos como escisión seguida o no de autoinjerto, dermoabrasión, láser decolorante pulsado, laser CO2 y erbium: YAG, éstos últimos con resultados promisorios. ${ }^{14,15,16}$

\section{Conalusión}

La enfermedad de Hailey-Hailey es una genodermatosis poco frecuente, que afecta de manera sustancial la calidad de vida del paciente. Su diagnóstico es difícil ya que simula otras patologías frecuentes, por lo tanto, se requiere de la sospecha diagnóstica asociada a una historia clínica completa y la histopatología que confirma el diagnóstico. El tratamiento es un desafío, debido a que no hay ninguna terapia específica ni totalmente efectiva. Sin embargo, es habitual que con evitar los agravantes y manejo tópico se pueda lograr una mejoría sustancial y los casos refractarios suelen ser la minoría. 


\section{REFERENCIAS}

1. Tchernev G, Cardoso K. Familial benign chronic pemphigus (HaileyHailey Disease): use of topical immunomodulators as a modern treatment option. Rev Med Chile 2011;139:633-637

2. Xing S, Wang Z, Liu S, Wu Y, Zhang S, Wang S. Three novel mutations of the ATP2C1 gene in Chinese families with HaileyHailey disease. J Eur Acad Dermatol Venereol.

3. Vaan Beek N, Patsatsi A, Gupta Y, Möller S, Freitag M, Lemcke S, et al. A Family with Atypical Hailey Hailey Disease- Is There More to the Underlying Genetics than ATP2C1? PLoS One. 2015 2;10(4)

4. Engin B, Kutlubay Z, Çelik U, Serdaroğlu S, Tüzün Y. HaileyHailey disease: A fold (intertriginous) dermatosis. Clin Dermatol. 2015;33:452-5

5. Gisondi P, Sampogna F, Annessi G, Girolomoni G. Severe impairment of quality of life in Hailey-Hailey disease. Acta Derm Venereol. 2005;85:132

6. Raegan H, O'Reilly K, Ralson J, Hideko K, Jerome S. Familial benign chronic pemphigus (Heiley-Haiey disease). Dermatology Online Journal. 2010;16:14

7. Ohata C. Hailey-Hailey disease. Cutis. 2014;8: 33-4

8. Le Saché-de Peufeilhoux L, Raynaud E, Bouchardeau A, Fraitag S, Bodemer C. Familial benign chronic pemphigus and doxycycline: a review of 6 cases. J Eur Acad Dermatol Venereol. 2014;28:370-373

9. Remission of refractory benign familial chronic pemphigus (haileyhailey disease) with the addition of systemic cyclosporine. Varada S, Ramirez-Fort MK, Argobi Y, Simkin AD J Cutan Med Surg. 2015;19:163
10. Ho D, Jagdeo J. Successful botulinum toxin (onabotulinumtoxinA) treatment of Hailey-Hailey disease.J Drugs Dermatol 2015:14(1):6870

11. Bagherani N, Smoller BR. The efficacy of botulinum toxin type A in the treatment of Hailey-Hailey disease. Dermatol Ther. 2016 Mar 11

12. Oral glycopyrrolate for the treatment of Hailey-Hailey disease. Kaniszewska M, Rovner R, Arshanapalli A, Tung R JAMA Dermatol. 2015;151(3):328

13. Graham PM, Melkonian A, Fivenson D. Familial benign chronic pemphigus (Hailey-Hailey disease) treated with electron beam radiation. JAAD Case Rep. 2016 16;2(2):159-61

14. Hunt KM, Jensen JD, Walsh SB, Helms ME, Soong VY, Jacobson ES, et al. Successful treatment of refractory Hailey-Hailey disease with a 595-nm pulsed dye laser: a series of 7 cases. J Am Acad Dermatol. 2015;72:735

15. Hochwalt PG, Christensen KN, Cantwell SR, Hocker TL, Otley CG, Brewer JD, et al. Carbon dioxide laser treatment for Hailey-Hailey disease: a retrospective chart review with patient-reported outcomes. Int J Dermatol. 2015; 54: 1309-14

16. Farahnik B, Blattner CM, Mortazie MB, Perry BM, Lear W, Elston DM. Interventional treatments for Hailey-Hailey disease. J Am Acad Dermatol. 2017; 76: 551- 558. 